4.5-year-old boy who had fallen chest first onto a barrel cactus. ${ }^{1}$ Several pediatric cases of intracardiac or intrapericardial penetration from a sewing needle have been reported. ${ }^{2-4}$ Several lessons should be learned from the present case. First, immediately after injury, children (or adults) may have minimal clinical signs or be symptom free when a foreign body is embedded in the myocardium or in the pericardial space. ${ }^{4}$ The time between penetration and diagnosis can take several hours. ${ }^{2}$ Second, radiography of the chest has usually failed to show any abnormality; most vegetal spines are radiolucent. Computed tomographic scanning has limitations in identifying small foreign bodies and objects with densities similar to surrounding structures. ${ }^{5}$ Third, a small thoracic puncture wound can hide potentially serious cardiac injuries. Finally, treatment options for cardiac foreign body or bodies have to be discussed case by case with cardiovascular surgeons. The presence of symptoms is a primary indication for surgery, especially in cases of cardiac tamponade, and median sternotomy is recommended if pericardiectomy is needed. ${ }^{4}$

\section{CONCLUSIONS}

Intracardiac vegetal spines or needles can be revealed by a 2-phase clinical evolution. This can lead to a delay in appropriate management in a life-threatening situation.

We gratefully acknowledge Mrs Claire Walker for her help in the translation of the manuscript.

\section{References}

1. O’Neill PJ, Sinha M, McArthur RA, Frechette A. Penetrating cactus spine injury to the mediastinum of a child. J Pediatr Surg. 2008;43:e33-5.

2. Perrotta S, Perrotta A, Lentini S. In patients with cardiac injuries caused by sewing needles is the surgical approach the recommended treatment? Interact Cardiovasc Thorac Surg. 2010;10:783-92.

3. Sola JE, Cateriano JH, Thompson WR, Neville HL. Pediatric penetrating cardiac injury from abuse: a case report. Pediatr Surg Int. 2008;24:495-7.

4. Actis Dato GM, Arslanian A, Di Marzio P, Filosso PL, Ruffini E. Posttraumatic and iatrogenic foreign bodies in the heart: report of fourteen cases and review of the literature. J Thorac Cardiovasc Surg. 2003;126:408-14.

5. Lydiatt DD, Hollins RR, Moyer DJ, Davis LF. Problems in evaluation of penetrating foreign bodies with computed tomography scans: report of cases. J Oral Maxillofac Surg. 1987;45:965-8.

\title{
Open chest core needle biopsy of left ventricle in the evaluation of suspected focal myocardial inflammation
}

\author{
Jukka Y. A. Lehtonen, MD, ${ }^{a}$ Janne J. Jokinen, MD, ${ }^{\mathrm{b}}$ Miia Holmström, MD,${ }^{\mathrm{c}}$ and Markku Kupari, MD, ${ }^{\mathrm{a}}$ \\ Helsinki, Finland
}

\begin{abstract}
Although clinical diagnosis is sufficient in most mild and moderate cases of myocarditis, histologic verification and classification by endomyocardial biopsy (EMB) are necessary if the clinical course is fulminant and when cardiac sarcoidosis (CS), giant cell myocarditis (GCM), or eosinophilic myocarditis is suspected. ${ }^{1}$ The well-known weakness of EMB is its limited sensitivity in patchy myocardial involvement. In our experience, a single EMB session detects at best 1 of 5 cases of $\mathrm{CS}^{2}$ and two thirds
\end{abstract}

\footnotetext{
From the Divisions of Cardiology ${ }^{\mathrm{a}}$ and Cardiac Surgery, ${ }^{\mathrm{b}}$ Heart and Lung Center, Helsinki, Finland; and HUS Radiology (Medical Imaging Center), ${ }^{\mathrm{c}}$ Helsinki University Central Hospital, Helsinki, Finland.

Disclosures: Authors have nothing to disclose with regard to commercial support.

Received for publication March 10, 2015; accepted for publication March 16, 2015; available ahead of print April 10, 2015

Address for reprints: Jukka Y. A. Lehtonen, MD, Helsinki University Hospital, Heart and Lung Center, PO Box 340, FI-00029 Helsinki, Finland (E-mail: jukka. lehtonen@hus.fi)

J Thorac Cardiovasc Surg 2015;149:e99-102

$0022-5223 / \$ 36.00$

Copyright (c) 2015 by The American Association for Thoracic Surgery

http://dx.doi.org/10.1016/j.jtcvs.2015.03.016
}

of cases of $\mathrm{GCM}^{3}$ even when targeted with the help of modern cardiac imaging. Biventricular biopsies may increase the diagnostic yield, ${ }^{4}$ as may the use of electrophysiologic mapping ${ }^{5}$ or intracardiac ultrasound ${ }^{6}$ to guide the sampling procedure. Unfortunately, despite repeated EMBs, the diagnosis may remain elusive. ${ }^{2}$

Surgical core needle myocardial biopsy through a left minithoracotomy provides several advantages over EMB in exchange for being more invasive, and therefore it is rarely used today. First and foremost, it gives access to areas of myocardium that are not reachable with a bioptome from inside the ventricular cavities. Further, instead of small endomyocardial samples, it provides full-thickness specimens of the left ventricular (LV) wall, and the possibility of visual inspection and palpation of the epicardial surface may help target the biopsy to areas of abnormal myocardium. We describe 3 patients with focal myocardial disease in whom histologic diagnosis was considered crucial for prognostic assessment and treatment. After multiple nondiagnostic EMBs, open surgical LV biopsy gave the diagnosis in each of them. 


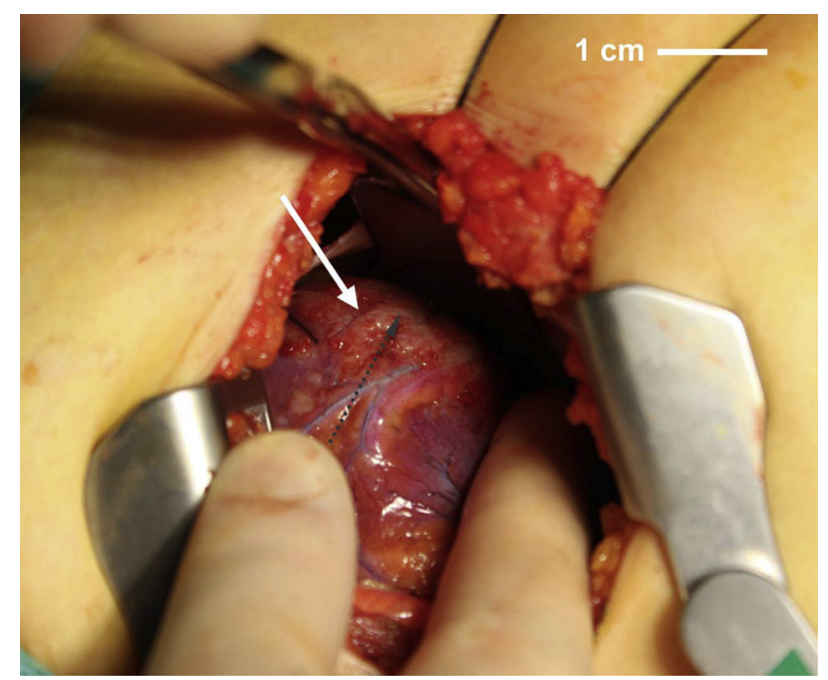

FIGURE 1. Visible and palpable abnormal area in the lateral aspect of the left ventricle (white arrow). Diagnostic biopsy specimen was taken by punching a $14 \mathrm{G}$ core needle through the lesion. Microscopy revealed granulomatous myocarditis consistent with CS (case 1).

\section{OPEN LEFT VENTRICULAR BIOPSY VIA MINITHORACOTOMY: THE TECHNIQUE}

The procedure is done under general anesthesia. A double-lumen endotracheal tube is used routinely because of the need for intermittent single-lung ventilation. An incision for anterolateral minithoracotomy $(<4 \mathrm{~cm})$ is placed in the sixth intercostal space at or just lateral to the midclavicular line. The pericardium is opened and suspended to provide good visibility and access to the apical, anterior, and lateral aspects of the left ventricle. Although the basal parts of the heart are not visualized directly, they can be palpated for any superficial abnormalities. We use the 14-gauge Gallini TZ (Gallini Medical Devices SRL, Mantova, Italy) disposable semiautomatic and spring-loaded biopsy instrument, for which the length of the core needle is adjustable from 10 to $20 \mathrm{~mm}$. The device provides specimens that include all layers of the left ventricle. The biopsy site is selected with the help of preoperative imaging studies and by intraoperative inspection and palpation of the LV wall (Figure 1). The biopsies are taken from visually abnormal areas and from the border zones between healthy and abnormal myocardium. Epicardial coronary arteries are carefully avoided. In addition to perpendicular fullthickness specimens, biopsies can be taken, with caution, parallel to the septum or the LV free wall. Frozen biopsy sections are sent to intraoperative microscopy to confirm the diagnostic adequacy of the samples. At the end, the biopsy sites are secured by meticulous suturing, and the pericardium is drained and closed loosely. The drain is removed when there is no longer active bleeding, usually on the first or second postoperative day.

\section{CASE 1}

A 51-year-old woman presented with acute-onset chest paint and ST-elevation in leads V1-2 of the electrocardiogram. She was admitted for primary percutaneous coronary intervention of an ST-elevation myocardial infarction. At angiography, the coronary arteries were fully patent, but there was segmental hypokinesia of the anterior LV wall. Intracoronary ultrasound did not show intimal tears in the left anterior descending branch, nor did acetylcholine induce visible coronary spasm. Troponin $\mathrm{T}$ was elevated at 269 $\mathrm{ng} / \mathrm{L}$ and gadolinium-enhanced cardiac magnetic resonance imaging (cMRI) revealed local hypokinesia, edema, and transmural late enhancement in the anterolateral LV wall (Figure 2,A). The patient was discharged with a diagnosis of myocarditis but was soon readmitted for monomorphic ventricular tachycardia. Cardiac 18-fluorodeoxyglucose position emission tomography $\left({ }^{18}\right.$ FDG-PET) was performed and showed signs of focal myocardial inflammation (Figure 2, B). Altogether, more than 30 EMB samples were taken from the left and right ventricles in 3 separate EMB sessions without any histologic abnormality. At minithoracotomy, the anterior LV wall appeared scarred. Full-thickness biopsies were taken and revealed necrosis, fibrosis, giant cells, and eosinophils consistent with GCM (Figure 2, C). Triple immunosuppression with cyclosporine, azathioprine, and prednisone was initiated and led to stabilization of LV function and control of arrhythmias.

\section{CASE 2}

An 18-year-old previously healthy woman presented with syncope due to a complete atrioventricular block. Initial transthoracic cardiac ultrasound revealed normal

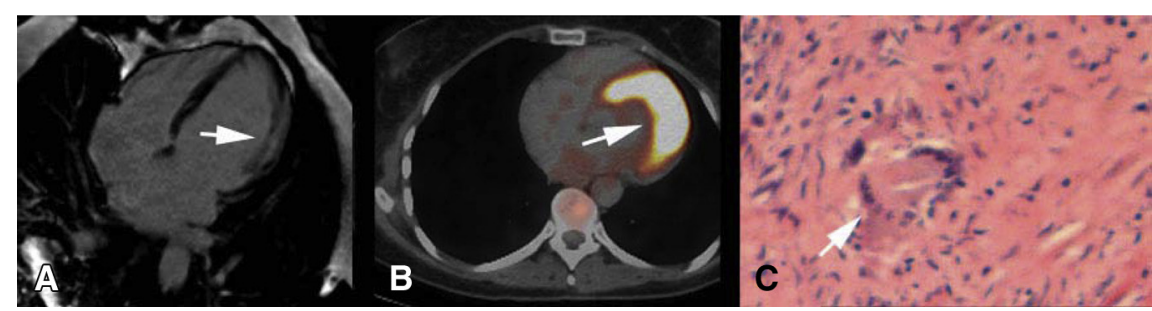

FIGURE 2. cMRI, ${ }^{18}$ FDG-PET, and histology in case 1 . A, Horizontal long-axis view of cMRI. Note myocardial late enhancement (arrow). B, ${ }^{18}$ FDG-PET shows a wide focus of active glucose uptake. C, Histology showing giant cells (arrow) and other findings diagnostic of GCM (40×, hematoxylin-eosin). 


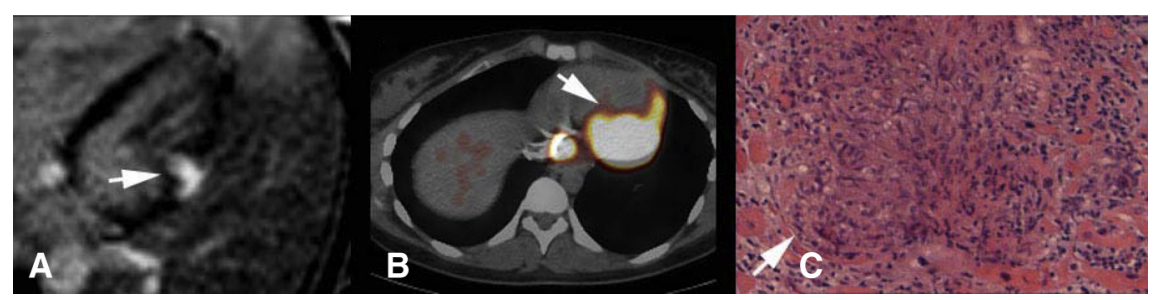

FIGURE 3. cMRI, ${ }^{18}$ FDG-PET, and histology in case 2. A, Horizontal long-axis view of cMRI showing a strongly late-enhancing local myocardial expansion (arrow). B, ${ }^{18}$ FDG-PET image with focal hot spot (arrow). C, Microscopic findings, including granulomas (arrow), consistent with CS (40×, hematoxylin-eosin).

LV dimensions and systolic function, but the posterolateral LV wall was unusually thick. An electrocardiogram-gated contrast computed tomography of the heart revealed 2 local intramyocardial expansions in the posterolateral LV wall. The expansions were strongly late enhancing at cMRI (Figure $3, A$ ) and showed increased uptake of ${ }^{18}$ FDG at PET (Figure 3, $B$ ), which also exposed spots of activity in the upper mediastinum, the mesenterium, and the parailiacal space. EMBs were taken from the left ventricle, targeting at the posterolateral wall expansion and, because of the atrioventricular block, from the right ventricular septal endocardium. All endomyocardial samples showed normal myocardium. At minithoracotomy, an area of the lateral LV wall was identified that that had an elevated and uneven surface. Full-thickness myocardial specimens revealed granulomatous inflammation consistent with sarcoidosis (Figure 3,C). Corticosteroid treatment was initiated. Later, a propensity to ventricular tachycardias developed in the patient, and her pacemaker, implanted because of the conduction block, was replaced with an intracardiac cardioverter-defibrillator.

\section{CASE 3}

A 29-year-old woman with mediastinal and pleural myxoid liposarcoma was referred for evaluation of an intramyocardial expansion found at routine postoperative computed tomography. A cMRI study showed a sharply demarcated late enhancing expansion in the lateral LV wall that measured $18 \times 16 \mathrm{~mm}$ and extended to the surface of the heart (Figure 4, A). At ${ }^{18}$ FDG-PET, both the myocardial expansion and some mediastinal lymph nodes had increased glucose uptake (Figure 4, B). Sampling of the mediastinal lymph nodes showed signs of granulomatous inflammation suggestive of sarcoidosis. However, there remained the concern of a solitary cardiac metastasis, and a further histologic study of the LV wall expansion was considered necessary. An EMB taken from inside the left ventricle was nonrevealing. Open surgical core needle biopsy was then performed and confirmed granulomatous inflammation consistent with sarcoidosis (Figure 4,C). Although the patient had no clinical manifestations of CS, corticosteroid therapy was initiated.

\section{DISCUSSION}

Current clinical guidelines detail the indications and technique of EMB, and acknowledge its limitations, but they do not discuss the role of open surgical myocardial biopsy in the evaluation of myocardial diseases. ${ }^{1}$ The present report was prepared to remind of the feasibility of open LV biopsy in the rare cases of myocardial disease in which histologic diagnosis is considered crucial, but all diagnostic attempts, including EMBs, have been nonrevealing. The importance of an exact diagnosis must be weighed carefully against the risks of the procedure, which, in addition to the risks of general anesthesia, include hemorrhage, cardiac tamponade, arrhythmias, infections, and postpericardiotomy syndrome. In theory, the procedure may damage the mitral valve apparatus and the intramural coronary artery branches. Because of the rarity of the open LV biopsy, there are no representative data of the

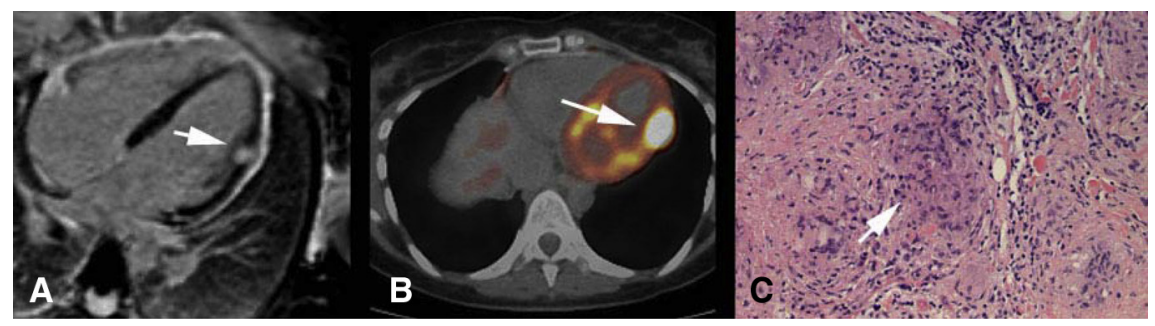

FIGURE 4. cMRI, ${ }^{18}$ FDG-PET, and histology in case 3. A, Horizontal long-axis view of gadolinium-MRI demonstrating a sharply demarcated late-enhancing expansion (arrow) in the lateral LV wall. B, ${ }^{18}$ FDG-PET image with hot spot in the LV lateral wall. C, Microscopic findings diagnostic of CS $(40 \times$, hematoxylin-eosin). 
rate of these complications in contemporary practice. Yet, the risks are likely to be lower than in the earlier fluoroscopy-assisted, closed transthoracic LV biopsies, which commonly led to pneumothorax and pleural reactions and resulted in cardiac tamponade in approximately $5 \%$ of the procedures. ${ }^{7}$

The introduction of cMRI and ${ }^{18}$ FDG-PET into routine diagnostic use has markedly facilitated the diagnosis of CS and increased the number of new diagnoses made in clinical practice. In Finland, the nationwide annual detection rate of clinically manifest CS is 20 to 30 times higher today than in the late 1990s. ${ }^{8}$ Although suspicions of CS arise more often than previously, verification of the disease remains challenging. This is because the diagnosis of CS necessitates histologic proof of sarcoidosis, preferably in the heart muscle or in extracardiac tissue if clinical manifestations and cardiac imaging are compatible with CS. ${ }^{9}$ Isolated CS, which is not an uncommon form of presentation in cardiology services, ${ }^{8,10}$ is particularly hard to diagnose with certainty. In our experience, repeated rounds of EMB may be needed until the bioptome hits an area diagnostic of $\mathrm{CS}^{2}{ }^{2}$ Whole-body PET may help in apparently isolated CS because it can uncover hidden extracardiac disease accessible for biopsy. ${ }^{2,8} \mathrm{GCM}$, which usually involves the heart muscle more diffusely than CS, also can manifest as a patchy myocardial inflammation escaping detection by EMB, as case 1 and our previous experience ${ }^{3}$ show.

\section{CONCLUSIONS}

A diagnosis of CS or GCM has important and long-lasting prognostic and therapeutic implications for the individual patient. Prednisone for CS and triple-drug immunosuppression for GCM are practically always indicated, and the threshold for implantation of a prophylactic intracardiac cardioverter-defibrillator decreases markedly. Yet, these treatments expose the patient to potentially serious side effects, which is why the diagnosis should leave no room for doubt. As a rule, therefore, CS- or GCM-specific treatment is unjustified on the basis of mere clinical suspicion but should rely on histologically confirmed disease. The present report shows how open LV biopsies can help clinicians solve diagnosis-resistant cases and adjust treatment to the underlying disease.

\section{References}

1. Cooper LT, Baughman KL, Feldman AM, Frustaci A, Jessup M, Kuhl U, et al The role of endomyocardial biopsy in the management of cardiovascular disease a scientific statement from the American Heart Association, the American College of Cardiology, and the European Society of Cardiology. Circulation. 2007; 116:2216-33.

2. Kandolin R, Lehtonen J, Graner M, Schildt J, Salmenkivi K, Kivistö SM, et al. Diagnosing isolated cardiac sarcoidosis. J Intern Med. 2011;270: 461-8.

3. Kandolin R, Lehtonen J, Salmenkivi K, Räisänen-Sokolowski A, Lommi J, Kupari M. Diagnosis, treatment, and outcome of giant-cell myocarditis in the era of combined immunosuppression. Circ Heart Fail. 2013;6:15-22.

4. Chimenti C, Frustaci A. Contribution and risks of left ventricular endomyocardial biopsy in patients with cardiomyopathies: a retrospective study over a 28-year period. Circulation. 2013;128:1531-7.

5. Lee JC, Seiler J, Blankstein R, Padera RF, Baughman KF, Tedrow UB. Cardiac sarcoidosis presenting as heart block. Circulation. 2009;120:1550-1.

6. Deshmukh A, Sewani A, Sachdeva R. Cardiac sarcoidosis masquerading as syncope with right ventricular septal mass. J Invasive Cardiol. 2012; 24:418-9.

7. Shirey EK, Hawk WA, Mukerji D, Effler DB. Percutaneous myocardial biopsy of the left ventricle: experience in 198 patients. Circulation. 1972;46:112-22.

8. Kandolin R, Lehtonen J, Airaksinen J, Vihinen T, Miettinen H, Ylitalo K, et al. Cardiac sarcoidosis: epidemiology, characteristics and outcome over 25 years in a nationwide study. Circulation. 2015;131:624-32.

9. Birnie DH, Sauer WH, Bogun F, Cooper JM, Culver DA, Duvernoy CS, et al. HRS consensus statement on the diagnosis and management of arrhythmias associated with cardiac sarcoidosis. Heart Rhythm. 2014;11:1305-23.

10. Okura Y, Dec GW, Hare JM, Kodama M, Berry GJ, Tazelaar HD, et al. Clinical and histopathologic comparison of cardiac sarcoidosis and idiopathic giant cell myocarditis. J Am Coll Cardiol. 2003;41:322-9. 